\title{
Closing the gap between business undergraduate education and the organisational environment: A Chilean case study applying experiential learning theory.
}

Leslier Valenzuela ${ }^{a}$, Oscar M. Jerez*a, Beatriz A. Hasbúnª, Verónica Pizarro ${ }^{\text {a }}$, Gabriela Valenzuela ${ }^{\mathrm{a}}$, César A. Orsini ${ }^{\mathrm{a}, \mathrm{b}}$

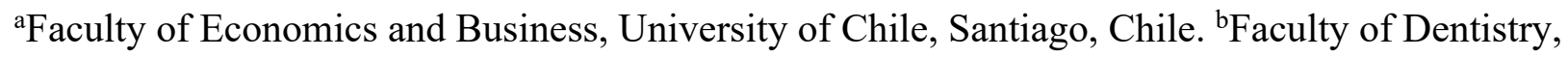
Universidad de los Andes, Chile.

*Corresponding author: Dr Oscar M. Jerez. Centro de Enseñanza y Aprendizaje, Facultad de Economía y Negocios, Universidad de Chile. Diagonal Paraguay 257, Piso -1, Torre 26. Santiago - Chile, Postcode: 8330015. E-mail: ojerez@fen.uchile.cl. Telephone:+56229772030

\section{Notes on contributors}

Leslier Valenzuela, MBA, holds a PhD in Marketing and is Professor of the Department of Business Administration, Faculty of Economics and Business, University of Chile.

Oscar M. Jerez, BPh, BE \& BE, MEd, EdD, is the Director of the Teaching and Learning Centre, Faculty of Economics and Business, University of Chile, and Consultant in Higher Education at LASPAU, affiliated with Harvard University.

Beatriz A. Hasbún, BASoc, MHD, is the executive Director at the Teaching and Learning Centre, Faculty of Economics and Business, University of Chile. She Participates in the Research Network of Chilean Education and the National Service-Learning Network.

Verónica Pizarro holds a Phd in Economics and Business Sciences from the Universidad Autónoma de Madrid in Spain. She is Professor of the Department of Management Control and Information Systems, Faculty of Economics and Business, University of Chile.

Gabriela Valenzuela is Business Administrator at the Faculty of Economics and Business, University of Chile and consultant in Learning Experience Design for the Chilean Government. 
Cesar A. Orsini, DDS, MEd, FHEA, RETF, is Lecturer and Researcher in Health Professions Education at the Faculty of Dentistry, Universidad de los Andes, Chile, and an Associate Research Fellow at the Teaching and Learning Centre, Faculty of Economics and Business, University of Chile. 


\title{
Closing the gap between business undergraduate education and the organisational environment: A Chilean case study applying experiential learning theory.
}

\begin{abstract}
In response to the continuous changes in Latin American higher education from transferonly knowledge towards the development of soft and specific skills, and the increasing demands for better-prepared professionals, the Learning Connected to the Organizational Environment method was introduced in the course of Marketing at the Faculty of Economics and Business of one public University in Chile. This was aimed as an integrated approach to education, providing pedagogical and social value by connecting organisations and real challenges with the learning objectives. This paper describes its design, implementation and initial impact on students' learning process. Results on the impact of the Learning Connected to the Organizational Environment method show that students valued learning with this new initiative $(n=158)$ and showed higher performance and improved quality of their written reports, along with higher evaluations of the teaching staff compared to students in the same course learning with traditional methods $(n=158)$. Discussion is centred on the value of this initiative and on suggestions for transference and future research.
\end{abstract}

Keywords: challenge-based learning; Chile; experiential learning theory; higher education; service learning; students performance. 


\section{Introduction}

Over the past decades Latin American higher education has experienced major changes, moving from a knowledge-acquisition focus towards the development of competencies that ensure students meet the skills and abilities demanded by the professional environment (Centro Nacional Tuning, 2004). These changes are partially explained by the increasing requirements from the productive sector and society, which demand better-prepared professionals. This has been of particular relevance for business and management education, where senior projects or capstone courses have increased in recent years aiming for students to work with real organisations and solve real professional problems (Heriot et al., 2008; Valenzuela \& Jerez, 2013)

As part of this trend, the Faculty of Business and Economics at one public University in Chile has progressively introduced experiential learning methods into the undergraduate curriculum. In this context, a recent initiative known as 'Learning Connected to the Organisational Environment' (LCOE) has been designed to bring Business Management students closer to real professional challenges.

Consequently, the purpose of this study is to describe the design and implementation, and report the preliminary impact of the LCOE as an example of such student-centred and experiential learning approach. After two years of pilot testing, where the programme was not fully integrated and therefore represented elective workload (Valenzuela \& Jerez, 2013), the LCOE was set to be the core learning activity in Marketing course. The aim of the LCOE initiative was to (1) improve students' sense of ownership in their learning process, (2) enable the integration of knowledge and experiences so to face real organisational challenges, (3) 
promote students' early approach to the work world, (4) enhance the learning outcomes through the development of generic and specific skills (Raza et al., 2011; De Miguel Díaz, 2005), and (5) improve students' satisfaction with the teaching on the aforementioned course.

The paper is organised as follows. In the next section, the design and implementation of the LCOE is described, along with its theoretical framework based on experiential learning theory. Next, the hypotheses by which the impact of the LCOE was tested are presented. These were focused on students' academic performance and the teaching evaluations, both of which were compared with a control or non-LCOE group. Additionally, the study set to assess the quality of students' reports presented to the participating organisations and students' satisfaction with the LCOE method. Afterwards, the methods applied to assess the impact of the LCOE are described. After presenting the findings, the final discussion is centred on what was learned from the project, offering several suggestions to those who seek to provide experiential learning education to business management students in similar contexts.

\section{Development and implementation of the LCOE}

The LCOE was designed in 2012, as an initiative to close the gap between business undergraduate education and the organisational environment responding to the university's quality assurance approach, in which students' graduate profile should be in line with the demands from the market and real organisations, and towards the enhancement of the linkage to the environment.

Three learning outcomes were designed for the course of Marketing, where the student was expected to: (1) diagnose an organisation's position within the industry and its potential 
short-to-middle and long-term sustainability, through model analyses that permitted the identification of critical success or failure factors for the organisation's marketing challenges; (2) develop solutions through the analysis of critical success factors identified for the strategic organisation study, with the aim of determining which are the most efficient and viable solutions for the short-, medium, and long-term; (3) elaborate an strategic marketing plan considering the organisation's short-, medium-, and long-term priorities to guide decision making rationale.

Until then, however, business management students in the course of Marketing developed their projects based on fictitious data and had no connection to real organisations. In an effort to better prepare students for business management practice, the LCOE initiative was designed for students to learn from and for professional contexts, based on the benefits reported by the introduction of capstone courses in different areas of higher education (Davis \& Comeau, 2004; Dutson et al., 1997). The LCOE was thought to provide an experiential learning activity in which the analytical knowledge gained from previous (and present) courses was joined with the practice of Business Management in a final, hands-on project. The LCOE and non-LCOE teaching and assessment approaches were identical, following the aforementioned learning outcomes, allowing a comparison between the two groups. The main difference was the real vs. simulated contact with organisations, i.e., fictitious data provided by lecturers.

At the beginning of semester, the University's Social Responsibility Unit invited several medium to large, public and private organisations to present their problems and challenges to students in the course of Marketing, which were related to the learning outcomes. Afterwards, students voluntarily chose two organisations they were interested to work with. The responsible teacher ensured that students were matched with the organisation and challenges they had chosen. Two teams of 5 to 6 students, known as student consultant teams (SCTs) were assigned 
to one organisation. Each student was therefore a member of one SCT that worked on solutions for the problems presented by the designed organisation. Each SCT developed one proposal, which represented the students' final report to be assessed in the Marketing course. The SCTs worked from the university premises, along with several visits to the organisations. Throughout the semester students learned through lectures and small group teaching, which informed the LCOE project. By the end of the semester the organisation representatives chose one of the two proposals. It was not uncommon that both proposals were chosen, complementing each other. Students received systematic follow-up and feedback during each of LCOE stages. Finally, there was a $360^{\circ}$ assessment of the proposals, which was independent of being chosen or not by the organisation.

The pedagogy underlying the LCOE is based on Kolb's (1984) model of experiential learning, which represents one of the most widely accepted approaches to understanding actionbased individual learning, linking education, work, and personal development by creating knowledge through the transformation of experience. This learning method has been referred to as an effective way for students to acquire and integrate knowledge from different courses with their personal traits and attitudes to solve real organisational challenges confronting business, governmental, and non-profit organisations (Shea et al., 2013). Therefore, the LCOE method took into account different aspects that facilitate learning, such as peer interactions, safe learning climates, students' commitment, and motivation (Orsini, Binnie \& Wilson, 2016).

What was not yet clear was the impact of the LCOE on the learning and teaching process. Consequently, four hypotheses were tested to measure its initial impact:

- Hypothesis 1: Students improve their performance in an LCOE-based course compared to a non-LCOE course with traditional teaching methods. 
- Hypothesis 2: Faculty teaching evaluations improve in an LCOE-based course compared to a non-LCOE course with traditional teaching methods.

- Hypothesis 3: SCTs prepare high-quality reports in an LCOE-based course compared to a non-LCOE course with traditional teaching methods

- Hypothesis 4: Students exhibit favourable perceptions of their learning and academic process in courses implementing the LCOE method. 


\section{Methods}

\section{Sample}

The study was conducted between August and December 2014 at the Faculty of Economics and Business at one public university in Chile. The Faculty's Research Ethics Committee reviewed and approved the study protocol. Students from all sections of the course of Marketing, applying the LCOE method, were invited to participate. This course corresponded to the fourth year of the Business Management curriculum. A randomly selected group of students that had taken the same course with the same faculty in a previous year, but without the LCOE method, were asked

for permission to access their academic performance, teacher evaluations, and written reports. The final number of students invited from this non-LCOE group was designed to match the numbers of the LCOE students. Additionally, faculty staff participating in those courses, i.e., teachers and teaching assistants, were asked for access to their end-of-semester evaluations.

All participation in the study was voluntary, with the option of withdrawing at any time with no consequences or explanations required, confidentiality and anonymity were guaranteed. A written informed consent was obtained before collecting any data.

\section{Variables and data collection}

Performance was measured by comparing students' grades in the course of Marketing for the LCOE and non-LCOE groups. In Chile, grading is based on a scale ranging from one (lowest) to seven (highest). 
LCOE students completed the Faculty of Economics and Business teaching evaluation surveys to monitor their perception of the teaching quality, which was compared to the teaching evaluations from the non-LCOE group. This survey measures student perceptions of different aspects of the course on a 7-point Likert scale, ranging from one (strongly disagree) to seven (strongly agree). Students' grades and teaching evaluations were accessed through the Instructor Management System.

To assess LCOE and non-LCOE students' final written reports, the learning outcomes identified in the syllabus were used to design an evaluation rubric. Each rubric included five key dimensions (Core knowledge, diagnosis or analysis, proposal creation, proposal development/design and recommendations/conclusions) with a maximum score of four for each one (Beginner, in process, competent, advanced). Reports from the non-LCOE group were reassessed as part of the study using the new rubric.

Finally, Students' perception of the LCOE learning and academic experience was measured adapting the teaching and learning assessment scale for business education. This instrument measures students' perceptions on 6 dimensions (teaching, learning environment, students' commitment, monitoring of learning, development of generic competencies, and learning experience with organisations) distributed in 18 categories, with a total of 47 items on a 5-point Likert scale ranging from zero (strongly disagree) to four (strongly agree). Previous research has reported the scale's construct validity and internal consistency (ranging from 0.708 to 0.828) (Olivares, Rodríguez, \& Salguero, 2011; Valenzuela \& Jerez, 2013). Sample items were: 'The course method has allowed me to develop important skills, which are beyond the lecture contents', 'Working with real organisations was crucial to achieve the learning outcomes', 'The experience with the organisations made me visualise my future work 
perspective'. Students answered this paper-based and self-administered survey towards the end of the semester.

\section{Data Analysis}

The software programme SPSS version 19.0 was used for our basic analyses. We computed descriptive statistics for all measures, and calculated the differences for the LCOE and nonLCOE groups on their written reports, performance, and teaching evaluations. Cohen's d measure for effect size was computed on the aforementioned differences. Additionally, a kmeans cluster analysis was conducted based on students' grades to assess how the LCOE influenced these different subgroups' performance. 


\section{Results}

The response rate of the LCOE student group was $87.7 \%$ (158/180), which included 63\% males and $37 \%$ females. The mean age was 22.6 (SD 3.26). Consequently, 158 students from a previous year from the course of Marketing were randomly invited to participate as part of the non-LCOE group. All 22 teachers agreed to participate and granted access to their end-ofsemester evaluations.

\section{Students' performance}

The mean performance score for the LCOE group was 5.38 (SD 0.43), whereas the nonLCOE group performance mean was of 4.99 (SD 0.29). The higher performance scores from the LCOE group represented a large effect size (Cohen's $d=1.06$ ). This shows that the LCOE method had a strong influence on student's academic performance, thus accepting hypothesis 1 .

Furthermore, the results obtained from the performance cluster analysis shown in Table 1 indicate that all LCOE groups, from low to high performances, scored higher that the non-LCOE groups. The medium and high performance group differences represented small and small-tomedium effect sizes, respectively. Interestingly, however, the medium-high, medium-low, and low performance group differences represented all medium effect sizes. In other words, the LCOE method proved to be of great benefit for lower-performance students. 


\section{Teachers' evaluations}

The mean evaluation score for the 22 Marketing course staff when teaching with traditional methods (i.e., non-LCOE) was of 5.03 (SD 1.56), however, when teaching the same course integrating the LCOE method their scores improved to a mean of 6.20 (SD 1.25). This difference represented a large effect size (Cohen's $\mathrm{d}=0.83$ ) and provided support for hypothesis 2.

\section{Quality of written reports}

As table 2 shows, all aspects assessed in the written report were higher in the LCOE group, hence accepting hypothesis 3. The project's diagnosis/analysis, proposal creation and development/design, and recommendations/conclusions represented all large effect sizes, indicating a strong influence of the LCOE in student's quality of written reports. The one exception where the effect size was very small was referred to the item of core knowledge,

\section{Perceptions of the LCOE method}

Table 3 presents students' perceptions of the learning process in the LCOE courses. Cronbach's alpha scores of internal consistency ranged from 0.745 to 0.816 . On the one hand, the dimensions with the highest scores were teaching, evaluation of the learning experience with the organisation, and learning environment. The teaching categories with the highest scores were instructors' professional expertise, followed by instructors' management of the teaching and 
learning processes, and commitment to students. The learning environment categories receiving the highest student scores were active learning and development of relevant work-related skills.

On the other hand, the lowest scores were exclusively student-related dimensions, such as perception and monitoring of learning, development of generic competencies, and student commitment. The categories with lowest scores corresponded to achievement and monitoring of learning outcomes, autonomous and responsible behavior and time management.

From this data, it is apparent that the main focus of LCOE, i.e., enhancing the teaching quality, working with organisations, and providing a safe learning climate, were highly endorsed by the students. There is still, however, work to be done in order to improve students' perceptions in key LCOE areas, such as self-regulated learning and the development of generic competencies. As such, hypothesis 4 was partially accepted.

\section{Discussion}

This study set out with the aim of describing the design and implementation of the LCOE method in a Marketing undergraduate course, and measuring its initial impact compared to the same course taught a with a non-LCOE and traditional lecture approach.

Hypothesis 1 was accepted as LCOE students performed largely better than the nonLCOE students. Moreover, one interesting finding was that the LCOE benefitted mostly lowerperformance students, indicating that this initiative has the potential to reduce the gap between higher- and lower-performing students. 
Students' evaluation of teachers was better in the LCOE than in the non-LCOE course, providing evidence to accept hypothesis 2 . This is coherent with previous studies reporting that faculty who use active methods to involve students in the learning process produce higher educational outcomes (Ala-Vähäla \& Saarinen, 2009; González \& Wagenaar, 2005; De Miguel Díaz, 2005).

Hypothesis 3 was accepted, as SCTs prepared higher-quality reports in the LCOE-based course than in the non-LCOE. It is interesting to note, however, that core knowledge represented a very small effect size. Thus, the LCOE and non-LCOE groups did not differ significantly in this aspect, which is reasonable as lectures and theoretical contents were taught similarly, and the mayor differences were showed in practical aspects were there was an influence of the real vs. simulated context.

Finally, hypothesis 4 was partially accepted, as students' perceived that the method contributed to the development of an effective teaching and learning environment, however, attributing greater importance to the teachers than to themselves. Therefore, it is important that future versions of the LCOE aim to transform students' perspectives by emphasising their role and responsibility in the learning process. This is in agreement with previous literature, which has highlighted the importance of students adopting an active role in the learning process (Devlin \& Samarawickrema, 2010; Marzano, 2007).

Although students reported favourable scores, especially in the dimension related to the experience of working with organisations, we detected room for improvement in the areas of student commitment and the development of generic competencies. These can be addressed by adding further practical activities, working closer with the organisations, and encouraging their autonomy. 
These results, while preliminary, provide further support for the LCOE method as a useful learning strategy for facilitating learning and community connections in business and professional development courses. Moreover, it provides a model for integrating prior academic and professional knowledge with personal traits and attitudes (Perrenoud, 2005), and allows students to experience key aspects of professional performance, such as competition and teamwork. This competition may have the potential to improve learning outcomes by enabling students to develop viable and creative proposals linked to real organisational problems.

The findings of this investigation complement those of earlier studies and add to a growing body of literature on the relevance of Experiential Learning Theory in business education (Dutson et al., 1997; Davis \& Comeau, 2004). This aims to provide students with the opportunity to integrate knowledge obtained from other and present courses during the development of applicable projects - and eventually develop practical skills and competencies.

Taken together, the LCOE experience suggests several courses of action for faculty who might transfer this (or similar) learning methods to their settings. In first place, active teaching and learning methods should be considered as a core element of the course. This contributes to the development of technical and generic competencies and promotes the development of students' interpersonal abilities (Devlin \& Samarawickrema, 2010). Prominent active learning methods include different experiential learning strategies, such as clinical methods and service learning, and their main components are the experience and the learning process itself that ultimately benefits both students and external organisations (Kolb, 1984).

In second place, student commitment should be highly encouraged by supporting persistence and willingness to participate in course activities to develop competencies and cognitive abilities (Marzano, 2007). In the LCOE method for instance, students adopted various 
organisational and decision-making strategies to achieve the desired academic outcomes. Student commitment can be evaluated by class attendance and participation, involvement in course activities, and compliance with deadlines (Walton \& Cohen, 2007).

Finally, it is of key importance to consider students' self-regulation and the teaching quality. Marzano, Pickering, and Heflebower (2011) claim that students should monitor their learning progress in terms of intrinsic factors, such as personal expectations, and extrinsic factors, such as the achievement of established goals. Students must also acknowledge their own responsibility for learning and the usefulness of what they have learned for future employment. This would enable them to better link theory with practice and identify challenges for their personal, educational, and professional development (Devlin \& Samarawickrema, 2010). The quality of teaching refers to faculty's pedagogic and personal activities that contribute to student learning and competency development, such as teachers' professional expertise, management of learning activities, commitment towards students, and networks with social and professional contacts (Bain, 2006; Marzano et al., 2011).

\section{Limitations and Future directions}

Despite the relevant findings, the scope and generalisability of this study was limited by the small sample coming from one Marketing course in one Chilean University, however, this was designed as an exploratory study on the LCOE method, and therefore subsequent studies are thought to report the implementation of LCOE and related experiential methods in a larger scale and involving other Business Management courses. Since students' perceptions of the teaching process were limited to quantitative methods and descriptive statistics, it was not possible to obtain students in-depth opinions about the LCOE. Notwithstanding, the initial impact of the 
LCOE was not limited to students' perceptions and was triangulated to evaluate the learning strategy from different sources.

A challenge for future research is to evaluate the extent to which the LCOE affects other variables, such as developing criteria for higher-performing SCTs; incorporating the external organisations' perceptions; ability to work with heterogeneous groups in diverse contexts and situations; the ability to act autonomously and responsibly in society; and the collection of qualitative data regarding students' feedback to provide an all-encompassing result if experiential learning actually works in enhancing the students' learning appreciation of the LCOE module.

This preliminary evaluation of the LCOE method has helped to shed light on how experiential learning strategies can be systematically incorporated into Chilean business education by providing the necessary support so to bring students closer to the organisational work-world and promote real-life learning experiences.

\section{References}

Ala-Vähäla, T., \& Saarinen, T. (2009). Building European-level Quality Assurance Structure: views from within ENQA. Quality in Higher Education, 15, 89-103.

Bain, K. (2006). Lo que hacen los mejores profesores universitarios [What the Best College Teachers Do]. Valencia: PUV.

Centro Nacional Tuning (2004). Proyecto Tuning- América Latina 2004-2006 [The Tuning Project - Latin America 2004-2006]. México: Centro Nacional Tuning. 
Davis, C. H., \& Comeau, J. (2004). Enterprise integration in business education: Design and outcomes of a capstone ERP-based undergraduate e-business management course. Journal of Information Systems Education, 15(3), 287.

De Miguel Díaz, M. (2005). Cambio de Paradigma Metodológico en la Educación Superior: Exigencias que conlleva [Changing the methodological paradigm in higher education: What is needed]. Cuadernos de Integración Europea, 2, 16-27.

Devlin, M., \& Samarawickrema, G. (2010). The criteria of effective teaching in a changing higher education context. Higher Education Research \& Development, 29, 111-124.

Dutson, A. J., Todd, R. H., Magleby, S. P., \& Sorensen, C. D. (1997). A Review of Literature on Teaching Engineering Design Through Project-Oriented Capstone Courses. Journal of Engineering Education, 86(1), 17-28.

González, J. \& Wagenaar, R. (2005). Tuning educational structures in Europe. Vol. 2. Universities' contribution to the Bologna Process. Bilbao, España: Publicaciones de la Universidad de Deusto.

Heriot, K., Cook, R. G., Simpson, L., \& Parker, R. (2008). The Use of Micro Student Consulting Projects as an Alternative to Traditional Field-Based Student Consulting Projects: An Exploratory Study. Journal of Entrepreneurship Education, 11, 59-74.

Kolb, D.A. (1984). Experiential learning: experience as the source of learning and development. Englewwood Cliffs, NJ: Prentice-Hall.

Marzano, R. J. (2007). The art and science of teaching: A comprehensive framework for effective instruction. Alexandria, VA: Association for Supervision and Curriculum Development.

Marzano, R. J., Pickering, D. J., \& Heflebower, T. (2011). The highly engaged classroom. Bloomington, IN: Marzano Research Laboratory. 
Olivares, P., Rodríguez, A. \& Salguero E. (2011). Evaluación integral del impacto de la metodología basada en desafíos profesionales y empresariales en la cátedra Marketing III [Integrated evaluation of the impact of methodology based on professional and businesses challenges in education]. Seminario para optar al título de Ingeniero Comercial. Chile: Universidad de Chile.

Orsini, C., Binnie, V. I., \& Wilson, S. L. (2016). Determinants and outcomes of motivation in health professions education: a systematic review based on self-determination theory. Journal of educational evaluation for health professions, 13. doi: 10.3352/jeehp.2016.13.19

Perrenoud, P. (2005) Diez nuevas competencias para enseñar [Ten new teaching competencies]. Educatio Siglo, Vol. 21, 23.

Raza, S. A., Hashmi, M. A., Zeeshan, A., Shaikh, F. M., \& Naqvi, A. H. (2011). Human and Social Capital Development for Self Efficacy of University Graduates: Bases for Development of Society. Asian Social Science, 7, 244-254.

Shea, E. R. O., Planas, J., Quan, M., \& Kazer, M. W. (2013). Service Learning Initiatives in Nursing Education. Journal of Catholic Higher Education, 32, 265-281.

Valenzuela, L., Jerez., O. (2013) Aprendizaje basado en los Desafíos Profesionales y Empresariales: Una Experiencia aplicada al Curso de Marketing [Learning based on professional and business challenges: Experiences applied to the Marketing course]. Revista Economía y Administración, 161, 60-67.

Walton, G. M., \& Cohen, G. L. (2007). A question of belonging: race, social fit, and achievement. Journal of Personality and Social Psychology, 92, 82-96. 
